\title{
Recycling Computed Answers in Rewrite Systems for Abduction*
}

\author{
Fangzhen Lin \\ Department of Computer Science \\ Hong Kong University of Science and Technology \\ Clear Water Bay, Kowloon, Hong Kong \\ Jia-Huai You \\ Department of Computing Science \\ University of Alberta \\ Edmonton, Alberta, Canada T6G 2E8
}

\begin{abstract}
In rule-based systems, goal-oriented computations correspond naturally to the possible ways that an observation may be explained. In some applications, we need to compute explanations for a series of observations with the same domain. The question whether previously computed answers can be recycled arises. A yes answer could result in substantial savings of repeated computations. For systems based on classic logic, the answer is yes. For nonmonotonic systems however, one tends to believe that the answer should be no, since recycling is a form of adding information. In this paper, we show that computed answers can always be recycled, in a nontrivial way, for the class of rewrite procedures proposed earlier in [12] for logic programs with negation. We present some experimental results on an encoding of the logistics domain.
\end{abstract}

${ }^{*}$ An extended abstract of parts of this paper appeared in the proceedings of IJCAI-03, Acapulco, Mexico. 


\section{Introduction}

The question we shall address in this paper is the following. With a sound and complete procedure for abduction, suppose we have computed explanations (conveniently represented as a disjunction) $E s=E_{1} \vee \ldots \vee E_{n}$ for observation $q$. Suppose also that in the course of computing explanations for another observation $p$, we run into $q$ again. Now, we may use the proofs $E s$ for $q$ without actually proving $q$ again. The question is this: will the use of the proofs $E s$ for $q$ in the proof for $p$ preserve the soundness and completeness of the procedure?

In this paper, we answer this question positively, but in a nontrivial way, for the class of rewrite procedures proposed in [12] for abduction in logic programming under (partial) stable model semantics ([7], [14]). The main result is a theorem (Theorem 4.7) that says recycling preserves the soundness and completeness.

The general idea of recycling is not new. Recycling in systems based on classic logic is always possible, since inferences in these systems can be viewed as transforming a logic theory to a logically equivalent one. In dynamic programming, it is the use of the answers for previously computed subgoals that reduces the computational complexity. In some game playing programs, for example in the world champion checker program Shinook (www.cs.ualberta.ca/ ${ }^{\sim}$ chinook), the endgame database stores the computed results for endgame situations which can be referenced in real-time efficiently.

However, the problem of recycling in a nonmonotonic proof system has rarely been investigated. We note that recycling is to use previous proofs. This differs from adding consequences. For example, it is known that the semantics based on answer sets or (maximal) partial stable models [4] do not possess the cautious nonmonotonicity property. That is, adding a consequence of a program could gain additional models thus losing some consequences. The following example is due to Dix [3]:

$$
P=\{a \leftarrow \operatorname{not} b . \quad b \leftarrow c, \operatorname{not} a . \quad c \leftarrow a .\}
$$

$P$ has only one answer set, $\{a, c\}$. Thus, $c$ is a consequence. When augmented with the rule $c \leftarrow$, the program gains a second answer set, $\{b, c\}$, and loses $a$ as a consequence.

Abduction in the framework of logic programming with answer sets [7] or partial stable models [14] has been studied extensively, and a number of formalisms and top-down query answering procedures have been proposed [1, 4, 5, 6, 9, 8, 10, 11, 12, 15, 16].

The class of rewrite procedures for abduction proposed in [12] is based on the idea of $a b$ duction as confluent and terminating rewriting. These systems are called canonical systems in the literature of rewrite systems [2]. The confluence and termination properties guaran- 
tee that rewriting terminates at a unique normal form independent of the order of rewriting. Thus, each particular strategy of rewriting yields a rewrite procedure.

These rewrite procedures can be used to compute explanations using a nonground program, under the condition that in each rule a variable that appears in the body must also appear in the head. Under this condition, an observation (a ground goal) is always rewritten to another ground goal, so that a rewriting mechanism desgined for ground programs works just as well. When the condition is not satisfied, one only needs to instantiate those variables that only appear in the body of a rule. For example, domain restricted programs [13] can be instantiated only on domain predicates for variables that do not appear in the head. This is a significant departure from the approaches that are based on ground computation where a function-free program is first instantiated to a ground program with which the intended models are then computed.

These rewrite procedures can also be used for answer set semantics in the following way. If a query $q$ is written into False, there cannot be any answer set containing $q$. This is because answer sets for normal programs are special cases of partial stable models. However, if the query is written into True, to see whether there is an answer set containing this query, one then only needs to check whether the context generated so far can be extended to an answer set, a task that is normally much easier than finding an answer set from scratch. There is a special case, however, when the corresponding propositional program is finite and so-called odd-loop free, partial stable models coincide with stable models. Thus the rewrite procedures are also sound and complete for these programs.

The next section defines logic program semantics. Section 3 reviews the rewriting framework. Then in Section 4 we formulate rewrite systems with computed rules and prove that recycling preserves soundness and completeness. Section 5 extends this result to rewrite systems with abduction, and Section 7 reports some experimental results.

\section{Logic Program Semantics}

A rule is of the form

$$
a \leftarrow b_{1}, \ldots, b_{m}, \text { not } c_{1}, \ldots, \operatorname{not} c_{n} .
$$

where $a, b_{i}$ and $c_{i}$ are atoms of the underlying propositional language $\mathcal{L}$. not $c_{i}$ are called default negations. A literal is an atom $\phi$ or its negation $\neg \phi$. A (normal) program is a finite set of rules.

The completion of a program $P$, denoted $\operatorname{Comp}(P)$, is a set of equivalences: for each atom $\phi \in \mathcal{L}$, if $\phi$ does not appear as the head of any rule in $P, \phi \leftrightarrow F \in \operatorname{Comp}(P)$; other- 
wise, $\phi \leftrightarrow B_{1} \vee \ldots \vee B_{n} \in C o m p(P)$ (with default negations replaced by the corresponding negative literals) if there are exactly $n$ rules $\phi \leftarrow B_{i} \in P$ with $\phi$ as the head. We write $T$ for $B_{i}$ if $B_{i}$ is empty.

The rewriting system of [12] is sound and complete w.r.t. the partial model semantics [14]. A simple way to define partial stable models without even introducing 3-valued logic is by the so called alternating fixpoints [17]. Let $P$ be a program and $S$ a set of default negations. Define a function over sets $S$ of default negations: $F_{P}(S)=\{\operatorname{not} a \mid P \cup S \forall a\}$. The relation $\vdash$ is the standard propositional derivation relation with each default negation not $\phi$ being treated as a named atom not $\_$.

A partial stable model $M$ is defined by a fixpoint of the function that applies $F_{P}$ twice, $F_{P}^{2}(S)=S$, while satisfying $S \subseteq F_{P}(S)$, in the following way: for any atom $\xi, \neg \xi \in M$ if not $\xi \in S, \xi \in M$ if $P \cup S \vdash \xi$, and $\xi$ is undefined otherwise. An answer set $E$ is defined by a fixpoint $S$ such that $F_{P}(S)=S$ and $E=\{\xi \in \mathcal{L} \mid P \cup S \vdash \xi\}$.

\section{Goal Rewrite Systems}

We introduce goal rewrite systems as formulated in [12].

A goal rewrite system is a rewrite system that consists of three types of rewrite rules: (1) Program rules from $\operatorname{Comp}(P)$ for literal rewriting; (2) Simplification rules to transform and simplify goals; and (3) Loop rules for handling loops.

A program rule is a completed definition $\phi \leftrightarrow B_{1} \vee \ldots \vee B_{n} \in \operatorname{Comp}(P)$ used from left to right: $\phi$ can be rewritten to $B_{1} \vee \ldots \vee B_{n}$, and $\neg \phi$ to $\neg B_{1} \wedge \ldots \wedge \neg B_{n}$. These are called literal rewriting.

A goal, also called a goal formula, is a formula which may involve $\neg, \vee$ and $\wedge$. A goal resulted from a literal rewriting from another goal is called a derived goal. Like a formula, a goal may be transformed to another goal without changing its semantics. This is carried out by simplification rules.

We assume that in all goals negation appears only in front of a literal. This can be achieved by simple transformations using the following rules: for any formulas $\Phi$ and $\Psi$,

$$
\begin{aligned}
& \neg \neg \Phi \rightarrow \Phi \\
& \neg(\Phi \vee \Psi) \rightarrow \neg \Phi \wedge \neg \Psi \\
& \neg(\Phi \wedge \Psi) \rightarrow \neg \Phi \vee \neg \Psi
\end{aligned}
$$




\subsection{Simplification rules}

The simplification rules constitute a nondeterministic transformation system formulated with a mechanism of loop handling in mind, which requires keeping track of literal sequences $g_{0}, \ldots, g_{n}$ where each $g_{i}, 0<i \leq n$, is in the goal formula resulted from rewriting $g_{i-1}$. Two central mechanisms in formalizing goal rewrite systems are rewrite chains and contexts.

- Rewrite Chain: Suppose a literal $l$ is written by its definition $\phi \leftrightarrow \Phi$ where $l=\phi$ or $l=\neg \phi$. Then, each literal $l^{\prime}$ in the derived goal is generated in order to prove $l$. This ancestor-descendant relation is denoted $l \prec l^{\prime}$. A sequence $l_{1} \prec \ldots \prec l_{n}$ is then called a rewrite chain, abbreviated as $l_{1} \prec^{+} l_{n}$.

- $\underline{\text { Context: }}$ A rewrite chain $g=g_{0} \prec g_{1} \prec \ldots \prec g_{n}=T$ records a set of literals $C=$ $\left\{g_{0}, \ldots, g_{n-1}\right\}$ for proving $g$. We will write $T\left(\left\{g_{0}, \ldots, g_{n-1}\right\}\right)$ and call $C$ a context. A context will also be used to maintain consistency: if $g$ can be proved via a conjunction, all of the conjuncts need be proved with contexts that are non-conflicting with each other. For simplicity, we assume that whenever $\neg F$ is generated, it is automatically replaced by $T(C)$, where $C$ is the set of literals on the corresponding rewrite chain, and $\neg T$ is automatically replaced by $F$.

Note that for any literal in a derived goal, the rewrite chain leading to it from a literal in the given goal is uniquely determined. As an example, suppose the completion of a program has the definitions: $a \leftrightarrow \neg b \wedge \neg c$ and $b \leftrightarrow q \vee \neg p$. Then, we get a rewrite sequence,

$$
a \rightarrow \neg b \wedge \neg c \rightarrow \neg q \wedge p \wedge \neg c .
$$

For the three literals in the last goal, we have rewrite chains from $a$ : $a \prec \neg b \prec \neg q ; a \prec$ $\neg b \prec p$; and $a \prec \neg c$.

Simplification Rules: Let $\Phi$ and $\Phi_{i}$ be goal formulas, $C$ be a context, and $l$ a literal.

SR1. $F \vee \Phi \rightarrow \Phi$

$\mathrm{SR} 1^{\prime} \Phi \vee F \rightarrow \Phi$

SR2. $F \wedge \Phi \rightarrow F$

SR2' $\Phi \wedge F \rightarrow F$

SR3. $T\left(C_{1}\right) \wedge T\left(C_{2}\right) \rightarrow T\left(C_{1} \cup C_{2}\right)$ if $C_{1} \cup C_{2}$ is consistent 
SR4. $T\left(C_{1}\right) \wedge T\left(C_{2}\right) \rightarrow F \quad$ if $C_{1} \cup C_{2}$ is inconsistent

SR5. $\Phi_{1} \wedge\left(\Phi_{2} \vee \Phi_{3}\right) \rightarrow\left(\Phi_{1} \wedge \Phi_{2}\right) \vee\left(\Phi_{1} \wedge \Phi_{3}\right)$

SR5'. $\left(\Phi_{1} \vee \Phi_{2}\right) \wedge \Phi_{3} \rightarrow\left(\Phi_{1} \wedge \Phi_{3}\right) \vee\left(\Phi_{2} \wedge \Phi_{3}\right)$

SR3 merges two contexts if they contain no complementary literals, otherwise SR4 makes it a failure to prove. SR4 can be implemented more efficiently by

$$
T(C) \wedge l \rightarrow F \quad \text { if } \neg l \in C
$$

Repeated applications of SR5 and SR5' can transform any goal formula to a disjunctive normal form (DNF).

\subsection{Loop rules}

After a literal $l$ is rewritten, it is possible that at some later stage either $l$ or $\neg l$ appears again in a goal on the same rewrite chain. Two rewrite rules are formulated to handle loops.

Definition 3.1 Let $S=l_{1} \prec^{+} l_{n}$ be a rewrite chain.

- If $\neg l_{1}=l_{n}$ or $l_{1}=\neg l_{n}$, then $S$ is called an odd loop.

- If $l_{1}=l_{n}$, then

- $S$ is called a positive loop if $l_{1}$ and $l_{n}$ are both atoms and each literal on $l_{1} \prec^{+} l_{n}$ is also an atom;

- $S$ is called a negative loop if $l_{1}$ and $l_{n}$ are both negative literals and each literal on $l_{1} \prec^{+} l_{n}$ is also negative;

- Otherwise, $S$ is called an even loop.

In all the cases above, $l_{n}$ is called a loop literal.

Loop Rules: Let $g_{1} \prec^{+} g_{n}$ be a rewrite chain.

LR1. $g_{n} \rightarrow F$

if $g_{i} \prec^{+} g_{n}$, for some $1 \leq i<n$, is a positive loop or an odd loop.

LR2. $g_{n} \rightarrow T\left(\left\{g_{1}, \ldots, g_{n}\right\}\right)$

if $g_{i} \prec^{+} g_{n}$, for some $1 \leq i<n$, is a negative loop or an even loop. 
A rewrite sequence is a sequence of zero or more rewrite steps $Q_{0} \rightarrow \ldots \rightarrow Q_{k}$, denoted $Q_{0} \rightarrow^{*} Q_{k}$, such that $Q_{0}$ is an initial goal, and for each $0 \leq i<k, Q_{i+1}$ is obtained from $Q_{i}$ by

- literal rewriting at a non-loop literal in $Q_{i}$, or

- applying a simplification rule to a subformula of $Q_{i}$, or

- applying a loop rule to a loop literal in $Q_{i}$.

Example 3.2 For the program given in the Introduction,

$$
P_{0}=\{a \leftarrow \operatorname{not} b . b \leftarrow c, \operatorname{not} a . c \leftarrow a .\}
$$

a is proved but b is not. This is shown by the following rewrite sequences:

$$
\begin{aligned}
& a \rightarrow \neg b \rightarrow \neg c \vee a \rightarrow \neg a \vee a \rightarrow F \vee a \rightarrow a \rightarrow T(\{a, \neg b\}) \\
& b \rightarrow c \wedge \neg a \rightarrow a \wedge \neg a \rightarrow \neg b \wedge \neg a \rightarrow F \wedge \neg a \rightarrow F
\end{aligned}
$$

Let $P_{1}=\{b \leftarrow \operatorname{not} c . c \leftarrow c\} .$.$b is proved and \neg b$ is not.

$$
b \rightarrow \neg c \rightarrow \neg c \rightarrow T(\{\neg b, \neg c\}) ; \quad \neg b \rightarrow c \rightarrow c \rightarrow F
$$

Note that, in general, the proof-theoretic meaning of a goal formula may not be the same as the logical meaning of the formula. For example, the goal formula $a \vee \neg a$ (a tautology in classic logic) could well lead to an $F$ if neither $a$ nor $\neg a$ can be proved, e.g., for the program $\{a \leftarrow \operatorname{not} a\}$.

Definition 3.3 A goal rewrite system for a program $P$ is a triple $\left\langle\mathcal{Q}_{L}, \mathcal{R}_{P}, \rightarrow\right\rangle$, where $\mathcal{Q}_{L}$ is the set of all goals, $\mathcal{R}_{P}$ is a set of rewrite rules which consists of program rules from Comp $(P)$, the simplification rules and the loop rules, and $\rightarrow$ is the set of all rewrite sequences. 


\subsection{Previous results}

Goal rewrite systems are like term rewriting systems [2] everywhere except at terminating steps: a terminating step at a subgoal may depend on the history of rewriting.

A set of rewrite sequences defines a binary relation, say $R$, on the set of goal formulas: $R\left(Q, Q^{\prime}\right)$ iff $Q \rightarrow^{*} Q^{\prime}$. Hence, a set of rewrite sequences corresponds to a binary relation.

Two desirable properties of rewrite systems are the properties of termination and confluence. Rewrite systems that possess both of these properties are called canonical systems. A canonical system guarantees that the final result of rewriting from any given goal is unique, independent of any order of rewriting.

Definition 3.4 A goal rewrite system $\left\langle\mathcal{Q}_{L}, \mathcal{R}_{P}, \rightarrow\right\rangle$ is terminating iff there exists no endless rewrite sequence $Q_{1} \rightarrow Q_{2} \rightarrow Q_{3} \rightarrow \ldots$. in $\rightarrow$.

Definition 3.5 A goal rewrite system $\left\langle\mathcal{Q}_{L}, \mathcal{R}_{P}, \rightarrow\right\rangle$ is confluent iff for any rewrite sequences $t_{1} \rightarrow^{*} t_{2}$ and $t_{1} \rightarrow^{*} t_{3}$, there exist $t_{4} \in \mathcal{Q}_{L}$ and rewrite sequences $t_{2} \rightarrow^{*} t_{4}$ and $t_{3} \rightarrow^{*} t_{4}$.

In [12], it is shown that all goal rewrite systems defined above are canonical, i.e., they are confluent and terminating. It was also shown any goal rewrite system is sound and complete w.r.t. the partial stable model semantics:

Theorem 3.6 Let $P$ be a finite program and $\left\langle\mathcal{Q}_{L}, \mathcal{R}_{P}, \rightarrow\right\rangle$ a goal rewrite system.

Soundness: For any literal $g$ and any rewrite sequence $g \rightarrow^{*} T\left(C_{1}\right) \vee \ldots \vee T\left(C_{m}\right)$, there exists a partial stable model $M_{i}$ of $P$, for each $i \in[1 . . m]$, such that $g \in C_{i} \subseteq M_{i}$.

Completeness: For any literal $g$ true in a partial stable model $M$ of $P$, there exists a rewrite sequence $g \rightarrow^{*} T\left(C_{1}\right) \vee \ldots \vee T\left(C_{m}\right)$ such that there exists $i \in[1 . . m], g \in C_{i} \subseteq M$.

\section{Goal Rewrite Systems with Computed Rules}

We first use two examples to illustrate the main technical results of this paper.

Example 4.1 Given a rewrite system $R^{0}$, suppose we have a rewrite sequence $\neg q \rightarrow a \rightarrow$ $a \rightarrow F$. The failure is due to a positive loop on a. We may recycle the computed answer by replacing the rewrite rule for $\neg q$ by the new rule, $\neg q \rightarrow F$. We thus get a new system, say $R^{1}$. Suppose in trying to prove $g$ we have

$$
g \rightarrow a \rightarrow \neg q \rightarrow F
$$


where the last step makes use of the computed answer for $\neg q$. The question arises as whether this way of using previously computed results guarantees the soundness and completeness. Theorem 4.7 to be proved later in this paper answers this question positively. To see it for this example, assume we have the following, successful proof in $R^{0}$

$$
g \rightarrow a \rightarrow \neg q \rightarrow a \rightarrow T(\{g, a, \neg q\})
$$

where the termination is due the even loop on a. Had such a sequence existed, recycling would have produced a wrong result. However, one can see that the existence of the rewrite step $a \rightarrow \neg q$ implies the existence of a different way to prove $\neg q$ :

$$
\neg q \rightarrow a \rightarrow \neg q \vee \ldots \rightarrow T(\{\neg q, a\}) \vee \ldots
$$

contradicting that $\neg q$ was rewritten to $F$ in $R^{0}$.

Before giving the next example, we introduce a different way to understand rewrite sequences. Since any goal formula can always be transformed to a DNF using the distributive rules SR5 and SR5', and the order of rewriting does not matter, we can view rewriting as generating a sequence of DNFs. Thus, a rewrite sequence in DNF from an initial goal $g$,

$$
g \rightarrow^{*} N_{1} \vee \ldots \vee N_{n}
$$

can be conveniently represented by derivation trees, or $d$-trees, one for each $N_{i}$ representing one possible way of proving $g$. For any $i$, the d-tree for $N_{i}$ has $g$ as its root node, wherein a branch from $g$ to a leaf node corresponds to a rewrite chain from $g$ that eventually ends with an $F$ or some $T(C)$. As such a disjunct is a conjunction, a successful proof requires each branch to succeed and the union of all resulting contexts to be consistent.

The next example is carefully constructed to illustrate that recycling may not yield the same answers as if no recycling were carried out. In particular, one can sometimes get additional answers.

Example 4.2 Consider the program:

$$
\begin{aligned}
& g \leftarrow a . \quad a \leftarrow \operatorname{not} b . \quad a \leftarrow e . \\
& b \leftarrow b . \quad e \leftarrow p . \quad p \leftarrow a .
\end{aligned}
$$

In Fig. $\square$ each d-tree consists of a single branch. The left two d-trees are expanded from 

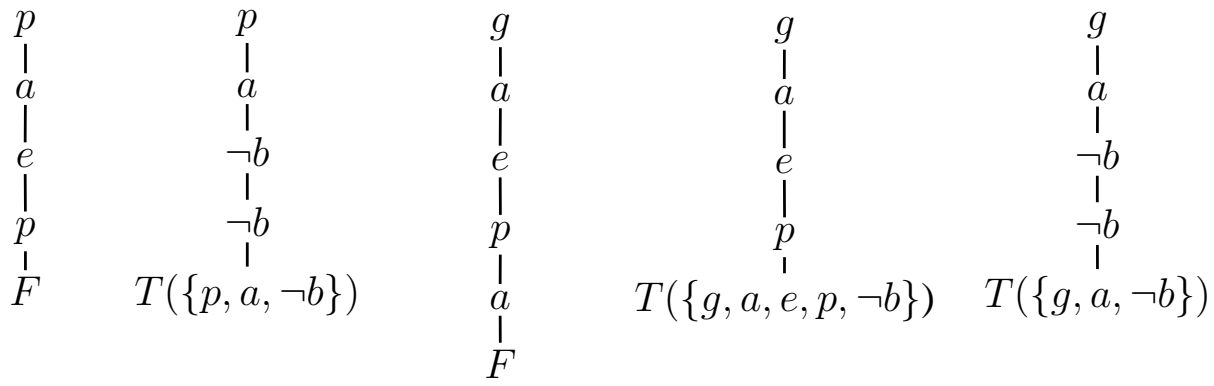

Figure 1: Recycling may generate extra proofs

goal p corresponding to the following rewrite sequence:

$$
p \rightarrow a \rightarrow e \vee \neg b \rightarrow p \vee \neg b \rightarrow F \vee \neg b \rightarrow \neg b \rightarrow \neg b \rightarrow T(\{p, a, \neg b\})
$$

The next two d-trees are for goal g, corresponding to the rewrite sequence:

$$
\begin{aligned}
& g \rightarrow a \rightarrow e \vee \neg b \rightarrow p \vee \neg b \rightarrow a \vee \neg b \\
& \rightarrow F \vee \neg b \rightarrow \neg b \rightarrow \neg b \rightarrow T(\{g, a, \neg b\})
\end{aligned}
$$

Now, we recycle the proof for $p$ in the proof for $g$ and compare it with the one without recycling. Clearly, the successful d-tree for $g$ (the fourth from the left) will still succeed as it doesn't involve any $p$. The focus is then on the d-tree in the middle, in particular, the node $p$ in it; this d-tree fails when no recycling was performed.

Since $p$ is previously proved with context $\{g, a, \neg b\}$, recycling of this proof amounts to terminating $p$ with a context which is the union of this context with the rewrite chain leading to $p$ (see the d-tree on the right). But this results in a successful proof that fails without recycling.

Though recycling appears to have generated a wrong result, one can verify that both generated contexts, $\{g, a, \neg b\}$ and $\{g, a, e, p, \neg b\}$, belong to the same partial stable model. Thus, recycling in this example didn't lead to an incorrect answer but generated a redundant one. Theorem 4.7 shows that this is not incidental. Indeed, if p is true in a partial stable model, by derivation (look at the d-tree in the middle), so must be e, a, and $g$.

\subsection{Rewrite systems with computed rules}

Given a goal rewrite system $R$, we may denote a rewrite sequence from a literal $g$ by $g \rightarrow_{R}$ E.

\section{Definition 4.3 (Computed rule)}

Let $R$ be a goal rewrite system in which literal $p$ is rewritten to its normal form. The computed rule for $p$ is defined as: If $p \rightarrow_{R} F$, the computed rule for $p$ is the rewrite rule 
$p \rightarrow F$; if $p \rightarrow_{R} T\left(C_{1}\right) \vee \ldots \vee T\left(C_{n}\right)$, then the computed rule for $p$ is the rewrite rule $p \rightarrow T\left(C_{1}\right) \vee \ldots \vee T\left(C_{n}\right)$.

For the purpose of recycling, a computed rule $p \rightarrow E$ is meant to replace the existing literal rewrite rule for $p$. If a computed rule $p \rightarrow F$ representing a failed derivation, it can be used directly as the literal rewrite rule for $p$. Otherwise, we must combine the contexts in $E$ with the rewrite chain leading to $p$, and keep only consistent ones.

\section{Recycling Rule:}

Let $g_{1} \prec^{+} g_{n}$ be a rewrite chain where $g_{n}$ is a non-loop literal. Let $G=\left\{g_{1}, \ldots, g_{n}\right\}$, and $g_{n} \rightarrow T\left(D_{1}\right) \vee \ldots \vee T\left(D_{k}\right)$ be the computed rule for $g_{n}$. Further, let $\left\{D_{1}^{\prime}, \ldots, D_{k^{\prime}}^{\prime}\right\}$ be the subset of $\left\{D_{1}, \ldots, D_{k}\right\}$ containing any $D_{i}$ such that $D_{i} \cup G$ is consistent. Then, the recycling rule for $g_{n}$ is defined as:

RC. $g_{n} \rightarrow T\left(G \cup D_{1}^{\prime}\right) \vee \ldots \vee T\left(G \cup D_{k^{\prime}}^{\prime}\right)$

Example 4.4 Consider the following program:

$$
\begin{array}{lll}
g \leftarrow a . & a \leftarrow p . & p \leftarrow \operatorname{not} a . \\
a \leftarrow \operatorname{not} p . & p \leftarrow \operatorname{not} b . & b \leftarrow \operatorname{not} a .
\end{array}
$$

and the proof:

$$
\begin{aligned}
& p \rightarrow \neg a \vee \neg b \rightarrow p \vee \neg b \rightarrow T(\{p, \neg a\}) \vee \neg b \\
& \rightarrow T(\{p, \neg a\}) \vee p \rightarrow T(\{p, \neg a\}) \vee T(\{p, \neg b\})
\end{aligned}
$$

We therefore have a computed rule for $p$ :

$$
p \rightarrow T(\{p, \neg a\}) \vee T(\{p, \neg b\})
$$

Now, in the course of proving $g$ we can recycle the computed rule for $p$ :

$$
g \rightarrow a \rightarrow p \vee \neg p \rightarrow T(\{g, a, p, \neg b\}) \vee p \rightarrow \ldots
$$

In the sequel, a rewrite system includes the recycling rule as well as zero or more computed rules. We note that the termination and confluence properties remain to hold for the extended systems.

We are interested in the soundness and completeness of a series of rewrite systems, each of which recycles computed answers generated on the previous one. For this purpose, given a program $P$ we use $R_{P}^{0}$ to denote the original goal rewrite system where literal rewrite rules are defined by the Clark completion of $P$. For all $i \geq 0, R_{P}^{i+1}$ is defined in terms of $R_{P}^{i}$ 

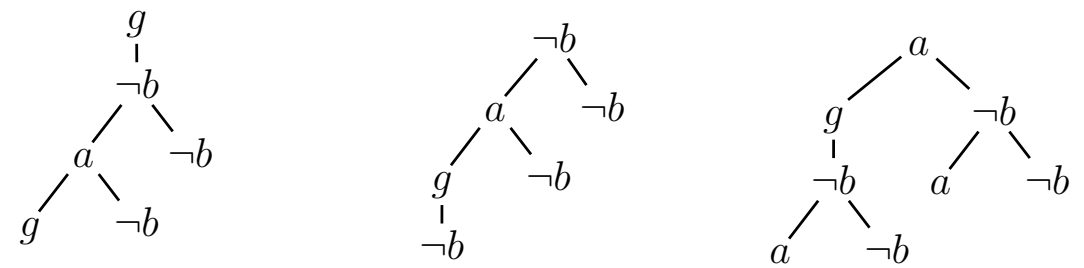

Figure 2: Loop rotation

as follows: Let $\Delta_{i}$ be the set of computed rules (generated) on $R_{P}^{i}$ for the set of literals $\mathcal{L}_{\Delta_{i}}$ Then, $R_{P}^{i+1}$ is the rewrite system obtained from $R_{P}^{i}$ by replacing the rewrite rules for the literals in $\mathcal{L}_{\Delta_{i}}$ by those in $\Delta_{i}$. In the rest of this sectin, we will always refer to a fixed program $P$. Thus we may drop the subscript $P$ and write $R^{i}$.

Definition 4.5 A rewrite system $R^{i}$ is sound iff, for any literal $g$ and rewrite sequence $g \rightarrow_{R^{i}}$ $T\left(C_{1}\right) \vee \ldots \vee T\left(C_{n}\right)$, and for each $C_{j}, j \in[1 . . n]$, there exists a partial stable model $M$ of $P$ such that $g \in C_{j} \subseteq M . R^{i}$ is complete iff, for any literal $g$ such that $g \in M$ for some partial stable model $M$ of $P$, there is a rewrite sequence $g \rightarrow_{R^{i}} T\left(C_{1}\right) \vee \ldots \vee T\left(C_{n}\right)$ such that for some $C_{j}, j \in[1 . . n], g \in C_{j} \subseteq M$.

An important property of provability by rewriting is the so-called loop rotation, which is needed in order to prove the completeness of recycling; namely, a proof (a successful branch in a d-tree) terminated by a loop rule can be captured in rotated forms.

To describe this property, we need the following notation about rewrite chains: Any direct dependency relation $l \prec l^{\prime}$ may be denoted by $l \cdot l^{\prime}$, and we allow a segment (which may be empty) of a rewrite chain to be denoted by a Greek letter such as $\delta, \theta$, and $\xi$. Thus, we may write $x \cdot \delta \cdot y$ to denote a rewrite chain from $x$ to $y$ via $\delta$, or $x \cdot \delta$ to mean a rewrite chain that begins with $x$ followed by the segment denoted by $\delta$. A rewrite chain may also be used to denote the set of the literals on it.

Lemma 4.6 (loop rotation)

Let $R^{0}$ be a rewrite system without computed rules. Let $\operatorname{Tr}$ be a d-tree for literal $g$ that succeeds with context $C$. Suppose a branch of Tr ends with a loop, $g \cdot \theta \cdot g$, for some $\theta$. Then, for any literal $l \in \theta$, there is a proof of $l$ that succeeds with the same context $C$.

Proof. A loop, $\pi=g \cdot l_{1} \cdot l_{2} \cdot \ldots \cdot l_{n} \cdot g$, where $g$ and $l_{i}$ are literals, can always be rotated as

$$
\begin{aligned}
& l_{1} \cdot l_{2} \cdot \ldots \cdot l_{n} \cdot g \cdot l_{1}, \\
& l_{2} \cdot \ldots \cdot l_{n} \cdot g \cdot l_{1} \cdot l_{2},
\end{aligned}
$$


and so on, so that if $\pi$ is a negative loop (or an even loop, resp.) so is its rotated loop. Rotation over a d-tree can be performed as follows: remove the top node $n$, and for any link from the top node, $n \cdot q$, attach the link $n \cdot q$ to any occurrence of $n$. The assumption of the existence of loop $g \cdot \theta \cdot g$ ensures that in every round of rotation there is at least one occurrence of the top node. (See Fig 2 for an illustration where rotation proceeds from left to right.) It can be seen that the type of a loop is always preserved and the set of literals on the tree remains unchanged.

\subsection{Soundness and completeness of recycling}

Below, given a literal $l$, by a proof of $l$ we mean a rewrite sequence from $l$ to $T\left(C_{1}\right) \vee \ldots \vee$ $T\left(C_{n}\right)$, where any $C_{i}$ can be referred to as a proof of $l$.

Theorem 4.7 For any $i \geq 0, R^{i}$ is sound and complete.

Proof. We prove the claim by induction on $i$. $R^{0}$, the system without computed rules, is sound and complete [12]. Now assume for all $j$ with $0 \leq j \leq i, R^{j}$ are sound and complete, and show that $R^{i+1}$ is also sound and complete.

We only need to consider the situations where rewriting in $R^{i+1}$ differs from that of $R^{i}$. Let $\mathcal{L}_{\Delta_{i}}$ be the set of literals whose computed rules are generated on $R^{i}$. We can first carry out rewriting without rewriting the literals that are in $\mathcal{L}_{\Delta_{i}}$. In this case, rewriting from $g$ in both $R^{i}$ and $R^{i+1}$ terminate at the same expression, which is either $F$ or a DNF, say $N_{1} \vee \ldots \vee N_{m}$. Each $N_{i}$ can be represented by a d-tree.

Soundness: Suppose $g \rightarrow_{R^{i+1}} T\left(D_{1}\right) \vee \ldots \vee T\left(D_{s}\right)$. For any $D \in\left\{D_{1}, \ldots, D_{s}\right\}$ we need to show that there is a partial stable model $M$ such that $D \subseteq M$. Consider the d-tree $\operatorname{Tr}$ that generates $D$ and suppose $g$ is its root node. We show inductively in a bottom-up fashion that all the literals on $\operatorname{Tr}$ must be in the same partial stable model. For any leaf node $p$ that is terminated by its computed rule

$$
p \rightarrow \ldots \vee T(C) \vee \ldots
$$

suppose $\operatorname{Tr}$ is the one that succeeded with context $C$. By the inductive hypothesis on $R^{j}$, we know that $R^{j}$ is sound for all $j \leq i$, thus there is a partial stable model $M$ such that $C \subseteq$ $D \subseteq M$. If a leaf node $q$ is terminated by a loop, by the loop rotation lemma (lemma 4.1), there is a proof of $q$ in $R^{i}$ using rotated loops. Otherwise we have an obvious case where a leaf node is rewritten to True by its Clark completion. 
In the inductive step, let $l_{1}, \ldots, l_{n}$ be the child nodes of some node $l$ and assume each $l_{i}$ is proved in $R^{i}$ hence in some partial stable model. We first show that they belong to the same partial stable model $M$. Then, we show that $l$ can also be proved in $R^{i}$ thus belonging to $M$ as well. Without loss of generality, assume there are only two child nodes: $l_{1} \rightarrow_{R^{i}}$ $T\left(Q_{1}\right) \vee \ldots \vee T\left(Q_{m}\right), l_{2} \rightarrow_{R^{i}} T\left(W_{1}\right) \vee \ldots \vee T\left(W_{n}\right)$. Since $D$ is constructed in $R^{i+1}$ using computed rules, by definitions of computed rule and the recycling rule, there are $Q_{i}$ and $W_{j}$ such that $Q_{i} \cup W_{j} \subseteq D$, and hence $Q_{i} \cup W_{j}$ is consistent. Then in $R^{i}$, the two contexts are merged by using simplification rule SR3, i.e.,

$$
l_{1} \wedge l_{2} \rightarrow_{R^{i}} \ldots \vee\left[T\left(Q_{i}\right) \wedge T\left(W_{j}\right)\right] \vee \ldots \rightarrow_{R^{i}} \ldots \vee T\left(Q_{i} \cup W_{j}\right) \vee \ldots
$$

Since $R^{i}$ is sound, there is a partial stable model $M$ such that $\left\{l_{1}, l_{2}\right\} \subseteq Q_{i} \cup W_{j} \subseteq M$. But $l$ is derivable from $l_{1}$ and $l_{2}$. Using the definition of partial stable models, it can be shown that $l$ must also be in $M$.

The induction allows us to conclude that for the top goal $g$ and its proof $D$ in $R^{i+1}$, we must have $g \in D \subseteq M$, for the same partial stable model $M$.

Completeness: We show that for any context generated in $R^{i}$, the same context will be generated in $R^{i+1}$. Then, $R^{i+1}$ is complete simply because $R^{i}$ is complete.

Let $p \in \mathcal{L}_{\Delta_{i}}$, and consider a proof of $g$ via $p$ and its d-tree. Since each branch of this d-tree can be expanded and eventually terminated independent of others, for simplicity, we consider a proof of $g$ simply by (an extension of) a branch $g \cdot \xi \cdot p$. In $R^{i+1}$ the computed rule for $p$ is used while in $R^{i}$ it is not. We only need to consider two cases of proof in $R^{i}$ : either $g$ is proved via $p$ and a previously computed rule, or the proof is terminated due to a loop.

(i) The case of loops. In expanding the rewrite chain $g \cdot \xi \cdot p$ in $R^{i}$, we may form a loop, say $g \cdot \xi \cdot p \cdot \xi^{\prime}$. If the loop is in $\xi^{\prime}$, exactly the same loop occurs in rewriting $p$ as the top goal in $R^{i}$, so it is part of the computed rule for $p$. Otherwise it is a loop that crosses over $p$, in the general form

$$
\pi=g \cdot \theta_{1} \cdot l \cdot \theta_{2} \cdot p \cdot \theta_{3} \cdot l
$$

where $l$ is the loop literal. As a special case of loop rotation over a branch (cf. Lemma4.1), the same way of terminating a rewrite chain presents itself in proving $p$ as the top goal in $R^{i}$, which is

$$
\pi^{\prime}=p \cdot \theta_{3} \cdot l \cdot \theta_{2} \cdot p .
$$

If the loop on $\pi$ is a negative loop (or an even loop, resp.), so is $\pi^{\prime}$. Thus the same context will be generated in $R^{i+1}$. 
(ii) $g$ is proved via $p$ and a previously computed rule. That is, $R^{i}$ gives a rewrite chain of the form $g \cdot \xi \cdot p \cdot \delta \cdot q$ where $q \rightarrow E$ is a computed rule generated on $R^{j}$ for some $j<i$. Suppose the context generated this way is $C$. Because of the existence of $p \cdot \delta \cdot q$, exactly the same computed rule $q \rightarrow E$ must be used in generating the computed rule for $p$ in $R^{i}$. It can be seen that the context generated in $R^{i+1}$ by recycling the computed answers for $p$ (which is computed via $q$ ) is exactly the same as the one that uses the computed answers for $q$ but not those for $p$. So, for any context generated this way in $R^{i}$, the same context will be generated in $R^{i+1}$ as well.

As given in the corollary below, if we only recycle failed proofs then exactly the same contexts will be generated.

Corollary 4.8 Let $R^{i}$ be a rewrite system where each computed rule is of the form $p \rightarrow F$. Let $g$ be a literal and $E$ be a normal form. Then, for any $i \geq 0, g \rightarrow_{R^{0}} E$ iff $g \rightarrow_{R^{i}} E$.

Proof. Let $\Delta$ be the set of literals whose rewrite rules are computed rules in $R^{i}$. Consider rewriting without rewriting on the literals in $\Delta$. Then, rewriting from $g$ terminates at the same expression $E^{\prime}$, which is either an $F$ or $T\left(C_{1}\right) \vee \ldots \vee T\left(C_{n}\right)$, in both $R^{0}$ and $R^{i}$. The claim then follows from the theorem above that for any $q \in \Delta, q \rightarrow_{R^{0}} F$ iff $q \rightarrow_{R^{i}} F$. That is, if $q \rightarrow_{R^{0}} F$, then $q$ is not in any partial stable model. The soundness of $R^{i}$ ensures that if $q \rightarrow_{R^{i}} Q$ where $Q \neq F$, then there is a partial stable model containing $q$, resulting in a contradiction. The converse is similar.

\section{$5 \quad$ Recycling in Abductive Rewrite Systems}

As shown in [12], the rewriting framework can be extended to abduction in a straightforward way: the only difference in the extended framework is that we do not apply the Clark completion to abducibles. That is, once an abducible appears in a goal, it will remain there unless it is eliminated by the simplification rule $S R 2$ or $S R 2^{\prime}$. In a similar way, the goal rewrite systems with computed rules in the previous section can be extended to abduction as well.

Definition 5.1 (Computed rule for abduction)

Let $R$ be an extended goal rewrite system for abduction. The computed rule for $p$ is defined as: If $p \rightarrow_{R} F$, the computed rule for $p$ is the rewrite rule $p \rightarrow F$; if

$$
\begin{gathered}
p \rightarrow_{R}\left[l_{11}\left(C_{11}\right) \wedge \cdots \wedge l_{1 k_{1}}\left(C_{1 k_{1}}\right)\right] \vee \ldots \vee \\
{\left[l_{m 1}\left(C_{m 1}\right) \wedge \cdots \wedge l_{m k_{m}}\left(C_{m k_{m}}\right)\right]}
\end{gathered}
$$


such that each $l_{i j}$ is either $T$ or an abducible literal, and $C_{i 1} \cup \cdots \cup C_{i k_{i}}$ is consistent for each $i$, then the computed rule for $p$ is the rewrite rule

$$
\begin{gathered}
p \rightarrow\left[l_{11}\left(C_{11}\right) \wedge \cdots \wedge l_{1 k_{1}}\left(C_{1 k_{1}}\right)\right] \vee \ldots \vee \\
{\left[l_{m 1}\left(C_{m 1}\right) \wedge \cdots \wedge l_{m k_{m}}\left(C_{m k_{m}}\right)\right]}
\end{gathered}
$$

\section{Recycling Rule:}

Let $g_{1} \prec^{+} g_{n} \prec p$ be a rewrite chain where $p$ is a non-loop literal. Let $G=\left\{g_{1}, \ldots, g_{n}, p\right\}$, and (2) be the computed rule for $p$. Then, the recycling rule for $p$ is defined as:

$\mathrm{RC}$.

$$
\begin{gathered}
p \rightarrow\left[l_{11}\left(C_{11} \cup G\right) \wedge \cdots \wedge l_{1 k_{1}}\left(C_{1 k_{1}} \cup G\right)\right] \vee \ldots \vee \\
{\left[l_{m 1}\left(C_{m 1} \cup G\right) \wedge \cdots \wedge l_{m k_{m}}\left(C_{m k_{m}} \cup G\right)\right]}
\end{gathered}
$$

\section{A Recycling Strategy}

We have shown that in theory, one can reuse the previously computed answers in our rewrite systems for abduction. To put the theory into practice, we need some effective strategies on how to recycle these computations.

If we want to compute the abduction of all goals in a set, without the framework of recycling introduced here, the only way is to compute them one by one independently. With the idea of recycling, we can try to recycle previously computed answers. The question is then which goals to compute first. This question arises even if we just want to compute the abduction of a single goal: instead of computing it using the original program, it may sometimes be better if we first compute the abduction of some other goals and recycle the results.

Assuming that goals are literals, a simple strategy for deciding the order of goals to be computed is to find out the dependency relations among the goals.

Definition 6.1 A literal $l$ is said to be depending on a literal $l^{\prime}$ if the atom in l depends on the atom in $l^{\prime}$. An atom $p$ is said to be depending on an atom $q$ if either $q$ is in the body of a rule whose head is $p$ or inductively, there is another atom $r$ such that $p$ depends on $r$, and $q$ is in the body of a rule whose head is $r$.

It is easy to see that if $l$ depends on $l^{\prime}$, but $l^{\prime}$ does not depend on $l$, then $l^{\prime}$ will never be sub-goaled to $l$ during rewriting, but $l$ could be sub-goaled to $l^{\prime}$. Thus if we need to compute the abduction of both $l$ and $l^{\prime}$, we should do it for $l^{\prime}$ first. 


\section{Experiments}

We have implemented a depth-first search rewrite procedure with branch and bound. The procedure can be used to compute explanations using a nonground program, under the condition that in each rule a variable that appears in the body must also appear in the head. When this condition is not satisfied, one only needs to instantiate those variables that only appear in the body of a rule. This is a significant departure from the approaches that are based on ground computation where a function-free program is first instantiated to a ground program with which the intended models are then computed.

To check the effectiveness of the idea of recycling, we tested our system on the logistics problem in [12]. This is a domain in which there is a truck and a package. A package can be in or outside a truck, and a truck can be moved from one location to another. The problem is that given state constraints such as that the truck and the package can each be at only one location at any given time, and that if the package is in the truck, then when the truck moves to a new location, so does the package, how we can derive a complete specification of the effects of the action of moving a truck from one location to another. Suppose that we have the following propositions: $\operatorname{ta}(x)(\mathrm{pa}(x))$ - the truck (package) is at location $x$ initially; in - the package is in the truck initially; $\operatorname{ta}(x, y, z)(\mathrm{pa}(x, y, z))$ - the truck (package) is at location $x$ after performing the action of moving it from $y$ to $z$; in $(y, z)$ - the package is in the truck after performing the action of moving the truck from $y$ to $z$. Then in [12], the problem is solved by computing the abduction of successor state propositions $\{\operatorname{ta}(x, y, z), \operatorname{pa}(x, y, z), \operatorname{in}(y, z)\}$ in terms of initial state propositions $\{\operatorname{ta}(x), \mathrm{pa}(x), \mathrm{in}\}$ (abducibles) using the following logic program:

$$
\begin{aligned}
& \operatorname{ta}(X, X 1, X) \\
& \operatorname{pa}(X, X 1, X 2) \leftarrow \operatorname{ta}(X, X 1, X 2), \operatorname{in}(X 1, X 2) \\
& \operatorname{ta}(X, X 1, X 2) \leftarrow X \neq X 2, \operatorname{ta}(X), \operatorname{not} \operatorname{taol}(X, X 1, X 2) . \\
& \operatorname{taol}(X, X 1, X 2) \leftarrow Y \neq X, \operatorname{ta}(Y, X 1, X 2) . \\
& \operatorname{pa}(X, X 1, X 2) \leftarrow \operatorname{pa}(X), \operatorname{not} \operatorname{paol}(X, X 1, X 2) \\
& \operatorname{paol}(X, X 1, X 2) \leftarrow Y \neq X, \operatorname{pa}(Y, X 1, X 2) \\
& \operatorname{in}(X, Y) \leftarrow \text { in. }
\end{aligned}
$$

Here the variables are to be instantiated over a domain of locations. For instance, given query

$\mathrm{pa}(3,2,3)$, our system would compute its abduction as $\mathrm{pa}(3) \vee \mathrm{in}$, meaning that for it to be true, either the package was initially at 3 or it was inside the truck. 


\begin{tabular}{|c|c|c||c|c|}
\hline \multirow{2}{*}{ Query } & \multicolumn{2}{|c||}{9 locations } & \multicolumn{2}{c|}{ 10 locations } \\
\cline { 2 - 5 } & NR & WR & NR & WR \\
\hline $\mathrm{pa}(1,2,3)$ & 0.71 & 0.41 & 1.50 & 0.89 \\
$-\mathrm{pa}(1,2,3)$ & 75.89 & 2.28 & 342.96 & 5.65 \\
$\mathrm{pa}(3,2,3)$ & 137.05 & 0.89 & 630.69 & 1.98 \\
$-\mathrm{pa}(3,2,3)$ & 2.97 & 1.98 & 7.64 & 5.03 \\
$\mathrm{pa}(1,5,7)$ & 122.87 & 0.75 & 278.07 & 1.31 \\
$-\mathrm{pa}(1,5,7)$ & 727.6 & 7.07 & 2534.09 & 19.08 \\
$\mathrm{pa}(7,5,1)$ & 108.66 & 17.82 & 188.50 & 30.72 \\
$-\mathrm{pa}(7,5,1)$ & 74.43 & 2.26 & 340.51 & 5.64 \\
$\mathrm{pa}(7,1,7)$ & 7619.72 & 20.78 & 29140.69 & 35.65 \\
$-\mathrm{pa}(7,1,7)$ & 2.98 & 2.01 & 7.71 & 5.05 \\
\hline
\end{tabular}

Table 1: Recycling in logistics domain. Legends: NR - no recycling; WR - recycling $\operatorname{ta}(X, Y, Z)$ goals. All times are in CPU seconds.

According to the definition in the last section, literals that contain $\mathrm{pa}(X, Y, Z)$ depend on those that contain in $(X, Y)$ and $\operatorname{ta}(X, Y, Z)$. But literals that contain in $(X, Y)$ and those that contain $\operatorname{ta}(X, Y, Z)$ do not depend on each other. So we should compute first the abduction of $\operatorname{in}(X, Y)$ and $\operatorname{ta}(X, Y, Z)$. Now $\operatorname{in}(X, Y)$ is solved by rule (9), $\operatorname{ta}(X, Y, X)$ by rule (3), and as it turned out, when $X \neq Z, \operatorname{ta}(X, Y, Z)$ is always false, and its computation is relatively easy. For instance, for the domain with 9 locations, query ta $(7,1,6)$ took only 2.6 seconds. In comparison, query pa $(7,1,7)$ took more than 7000 seconds without recycling.

Table 1 contains run time data for some representative queries. ${ }^{1}$ For comparison purpose, each query is given two entries: the one under "NR" refers to regular rewriting system without using recycling, and the one under "WR" refers to rewriting system using computed rules about $\operatorname{ta}(X, Y, Z)$. As one can see, especially for hard queries like pa $(7,1,7)$, recycling in this case significantly speeds up the computation.

\footnotetext{
${ }^{1}$ Our implementation was written in Sicstus Prolog, and the experiments were done on a PIII $1 \mathrm{GHz}$ notebook with $512 \mathrm{MB}$ memory. For generating explanations for regular rewriting system, our implementation is a significant improvement over the one in [12]. For instance, for a domain with 7 locations query pa(3,2,3) took more than 20 minutes for the implementation reported in [12], but required less than 1 second under our implementation running on a comparable machine.
} 


\section{Concluding remarks and future work}

We have considered the problem of how to reuse previously computed results for answering other queries in the abductive rewriting system of Lin and You [12] for logic programs with negation, and showed that this can indeed be done. We have also described a methodology of using the recycling system in practice by analysing the dependency relationship among propositions in a logic programs. We applied this methodology to the problem of computing the effect of actions in a logistics domain, the same one considered in [12], and our experimental results showed that recycling in this domain can indeed result in good performance gain.

For future work we are looking for more domains to try our system on and to implement a system that can automatically analyse a program and decide how best to recycle previous computations.

\section{References}

[1] L. Console, D. Theseider, and P. Porasso. On the relationship between abduction and deduction. J. Logic Programming, 2(5):661-690, 1991.

[2] N. Dershowitz and P. Jouannaud. Rewrite systems. In Handbook of Theoretical Computer Science, Vol B: Formal Methods and Semantics, pages 243-320. North-Holland, 1990.

[3] J. Dix. Classifying semantics of logic programs. In Proc. First Workshop on LPNMR, pages 167-180, 1991.

[4] P. Dung. Negations as hypothesis: An abductive foundation for logic programming. In K. Furukawa, editor, 8th ICLP. MIT press, 1991.

[5] P. Dung. An argumentation theoretic foundation for logic programming. J. Logic Programming, 22:151-177, 1995.

[6] K. Eshghi and R. Kowalski. Abduction compared with negation by failure. In Proc. 6th Int'l Conference on Logic Programming, pages 234-254. MIT Press, 1989.

[7] M. Gelfond and V. Lifschitz. The stable model semantics for logic programming. In Proc. 5th Int'l Conference on Logic Programming, pages 1070-1080. MIT Press, 1988. 
[8] A. Kakas, R. Kowalski, and F. Toni. The role of abduction in logic programming. In Handbook of Logic in Artificial Intelligence and Logic Programming. Oxford University, 1995.

[9] A. Kakas and P. Mancarella. Generalized stable models: a semantics for abduction. In Proc. 9th European Conference on Artificial Intelligence, pages 285-291, 1990.

[10] A. Kakas and P. Mancarella. On the relation between truth maintenance and abduction. In Proc. PRICAI'90, pages 438-443, 1990.

[11] A. Kakas, A. Michael, and C. Mourlas. ACLP: abductive constraint logic programming. J. Logic Programming, 44(1-3):129-178, 2000.

[12] F. Lin and J. You. Abduction in logic programming: a new definition and an abductive procedure based on rewriting. In Proc. IJCAI'01, pages 655-661, 2001.

[13] I. Niemelä. Logic programs with stable model semantics as a constraint programming paradigm. Annual of Mathematics and Artificial Intelligence, 25(3-4):241-273, 1999.

[14] T. Przymusinski. Extended stable semantics for normal and disjunctive logic programs. In Proc. 7th ICLP, pages 459-477. MIT Press, 1990.

[15] K. Satoh and N. Iwayama. Computing abduction using the TMS. In Proc. 8th Int'l Conference on Logic Programming, pages 505-518, 1991.

[16] K. Satoh and R. Iwayama. A query evaluation method for abductive logic programming. In Proc. Joint Int'l Conference and Symposium on Logic Programming, pages 671-685, 1992.

[17] J. You and L. Yuan. On the equivalence of semantics for normal logic programs. JLP, 22:212-221, 1995. 